\title{
BOUNDED TRUNCATION ERROR FOR LONG-RUN AVERAGES IN INFINITE MARKOV CHAINS
}

\author{
HENDRIK BAUMANN, ${ }^{*}$ Clausthal University of Technology \\ WERNER SANDMANN, ${ }^{* *}$ University of Derby
}

\begin{abstract}
We consider long-run averages of additive functionals on infinite discrete-state Markov chains, either continuous or discrete in time. Special cases include long-run average costs or rewards, stationary moments of the components of ergodic multi-dimensional Markov chains, queueing network performance measures, and many others. By exploiting FosterLyapunov-type criteria involving drift conditions for the finiteness of long-run averages we determine suitable finite subsets of the state space such that the truncation error is bounded. Illustrative examples demonstrate the application of this method.
\end{abstract}

Keywords: Infinite Markov chain; additive functional; long-run average; state space truncation; bounded truncation error; Foster-Lyapunov-type criterion; drift condition

2010 Mathematics Subject Classification: Primary 60J22

Secondary 60J10;60J27;60J28

\section{Introduction}

We consider infinite Markov chains, either continuous or discrete in time, on a countable state space 8 . In continuous time we denote the Markov chain by $\left(X_{t}\right)_{t \geq 0}$ and its generator matrix by $\boldsymbol{Q}=\left(q_{i j}\right)_{i, j \in \mathcal{s}}$. In discrete time we denote the Markov chain by $\left(Y_{n}\right)_{n \in \mathbb{N}}$ and its transition probability matrix by $\boldsymbol{P}=\left(p_{i j}\right)_{i, j \in \varsigma \text {. }}$

For irreducible recurrent Markov chains, an invariant measure $\psi=\left(\psi_{i}\right)_{i \in s}$ exists, which is unique up to a multiplicative constant, and for $f^{(1)}, f^{(2)}: s \rightarrow \mathbb{R}$ with $\psi\left|f^{(1)}, \psi\right| f^{(2)} \mid<\infty$, we have

$$
\lim _{t \rightarrow \infty} \frac{\int_{0}^{t} f^{(1)}\left(X_{s}\right) \mathrm{d} s}{\int_{0}^{t} f^{(2)}\left(X_{S}\right) \mathrm{d} s}=\frac{\psi f^{(1)}}{\psi f^{(2)}}, \quad \text { respectively } \quad \lim _{N \rightarrow \infty} \frac{\sum_{n=0}^{N} f^{(1)}\left(Y_{n}\right)}{\sum_{n=0}^{N} f^{(2)}\left(Y_{n}\right)}=\frac{\psi f^{(1)}}{\psi f^{(2)}}
$$

with probability 1 ; see [8, pp. 85-86, 203-209]. Hence, obtaining $\psi f$ for functions $f$ on the state space of irreducible recurrent Markov chains is of high practical relevance.

Particularly important special cases of irreducible recurrent Markov chains are ergodic Markov chains, where $\psi$ is the unique stationary distribution $\pi=\left(\pi_{i}\right)_{i \in S}$ that coincides with the limiting distribution. It satisfies $\pi \boldsymbol{Q}=0$ in continuous time and $\pi \boldsymbol{P}=\pi$ in discrete time, respectively. According to the respective ergodic theorems, if the expectation $\mathbb{E}_{\pi}[|f|]$ for a function $f: s \rightarrow \mathbb{R}$ is finite, then the averages of additive functionals converge (for time approaching infinity) almost surely to the stationary expectation $\mathbb{E}_{\pi}[f]=\sum_{i \in \S} \pi_{i} f(i)=\pi f$,

Received 7 November 2012; revision received 23 July 2014.

* Postal address: Department of Applied Stochastics and Operations Research, Clausthal University of Technology, Erzstr. 1, D-38678 Clausthal-Zellerfeld, Germany. Email address: hendrik.baumann@tu-clausthal.de

** Postal address: School of Computing and Mathematics, University of Derby, Kedleston Road, Derby DE22 1GB, UK. 
that is,

$$
\lim _{t \rightarrow \infty} \frac{1}{t} \int_{0}^{t} f\left(X_{s}\right) \mathrm{d} s=\mathbb{E}_{\pi}[f], \quad \text { respectively } \lim _{N \rightarrow \infty} \frac{1}{N} \sum_{n=0}^{N} f\left(Y_{n}\right)=\mathbb{E}_{\pi}[f]
$$

with probability 1 ; see [2, pp. 52-54], [19, pp. 264-265] for the continuous-time case, and [2, pp. 16-19], [19, pp. 45-47] for the discrete-time case. Hence, $\mathbb{E}_{\pi}[f]$ is the long-run average of an additive functional on the respective Markov chain.

We are interested in approximating $\psi f$ for infinite recurrent Markov chains by using finitestate truncations, which is important in cases where no analytical solution to the infinite chain is available and the state space must be truncated, e.g. for computational purposes. While corresponding truncation approximations of stationary distributions have been studied quite extensively, see [9], [11], [13], [14], [16], [24], [25], and [18, Chapter 7], there is a lack of similar studies for $\psi f$ or its special case of stationary expectations.

The goal is to perform the truncation such that the truncation error is bounded by an a priori specified constant. Obviously, since in general no information on the value of $\psi f$ is available in advance we have to bound the relative truncation error. Therefore, provided that $\psi f$ is finite, we shall provide a method for determining a finite subset $\mathcal{C} \subset \&$ of the state space such that for a small prescribed $\varepsilon \in(0,1)$,

$$
\frac{\sum_{i \in \mathcal{C}} \psi_{i} f(i)}{\sum_{i \in \delta} \psi_{i} f(i)} \geq 1-\varepsilon
$$

Note that this yields a 'true' a priori truncation error bound in that $\varepsilon$ indeed bounds the proportion of $\psi f$ that is cut off by the finite-state truncation. There is no need to compute the left-hand side, in particular the numerator, of the inequality (1) since we shall guarantee that $\mathcal{C}$ is chosen such that the truncation error is bounded by $\varepsilon$. In other words, we do not aim to compute the truncation error a posteriori, but we start with an a priori fixed maximum truncation error and obtain a suitable truncation.

In Section 2 we establish appropriate 'Foster-Lyapunov-type criteria' involving 'drift conditions' and in Section 3 we show how to use them for determining appropriate finite sets $\mathcal{C} \subset \&$ that meet (1). Subsequently, in Section 4 we provide application examples. Finally, in Section 5 we conclude the paper and outline further research directions.

\section{Foster-Lyapunov-type criteria}

For discrete-time Markov chains $\left(Y_{n}\right)_{n \in \mathbb{N}}$ the drift function $d_{g}: \& \rightarrow \mathbb{R}$ with respect to a function $g: \delta \rightarrow \mathbb{R}$ is defined by

$$
d_{g}(i)=\mathbb{E}\left[g\left(Y_{n}\right)-g\left(Y_{n-1}\right) \mid Y_{n-1}=i\right]=\sum_{j \in \S} p_{i j} g(j)-g(i),
$$

that is, when writing $g$ and $d_{g}$ in the form of column vectors, $d_{g}=\boldsymbol{P} g-g$. Hence, $d_{g}(i)$ is the (generalized) drift in state $i$ with respect to $g$.

For continuous-time Markov chains $\left(X_{t}\right)_{t \geq 0}$ the drift function $d_{g}: s \rightarrow \mathbb{R}$ with respect to a function $g: \& \rightarrow \mathbb{R}$ is defined by

$$
d_{g}(i)=\frac{\mathrm{d}}{\mathrm{d} t} \mathbb{E}\left[g\left(X_{t}\right) \mid X_{t}=i\right]=\sum_{j \in \delta} q_{i j} g(j),
$$


that is, when writing $g$ and $d_{g}$ in the form of column vectors, we have $d_{g}=\boldsymbol{Q} g$.

For finite $\mathcal{C} \subset \&, \gamma>0$ and $f, g: \& \rightarrow \mathbb{R}_{\geq 0}$ we consider the following conditions:

(C1) for all $i \in \delta \backslash \mathcal{C}: d_{g}(i) \leq-\gamma f(i)$;

(C2) for all $i \in \mathcal{C}: d_{g}(i)<\infty$;

(C3) for all $r<\infty:|\{i \in \&: g(i) \leq r\}|<\infty$.

Conditions of this form are often referred to as Foster-Lyapunov-type criteria since they are generalizations of classical criteria for positive recurrence or ergodicity, respectively, of Markov chains. For discrete-time Markov chains, in the special case where $f(i)=1$, we have a criterion for positive recurrence, which in fact is very famous. In the case $|\mathcal{C}|=1$ it is due to Foster [10], in the slightly more general case of arbitrary finite $\mathcal{C}$ it was proven by Pakes [17]. For continuous-time Markov chains, in the special case where $f(i)=1$, we have a famous criterion for regularity and positive recurrence, which is due to Tweedie [21, Theorem 2.3]. Appropriate functions $g$ with respect to which the drift function $d_{g}$ is defined are often called Lyapunov functions and the conditions on $d_{g}$ as (generalized) drift conditions.

Theorem 1. Let $\left(Y_{n}\right)_{n \in \mathbb{N}}$ be an irreducible discrete-time Markov chain with transition probabilitymatrix $\boldsymbol{P}=\left(p_{i j}\right)_{i, j \in s,} \mathcal{C} \subset$ s finite $\gamma>0$ and let $f, g: \& \rightarrow \mathbb{R}_{\geq 0}$ meet the conditions $(C 1)$ (C3). Then $\left(Y_{n}\right)_{n \in \mathbb{N}}$ is recurrent and for any invariant measure $\psi$ the sum $\psi f=\sum_{i \in s} \psi_{i} f(i)$ is finite.

The recurrence of $\left(Y_{n}\right)_{n \in \mathbb{N}}$ follows from [21, Theorem 3.3] so that an invariant measure $\psi$ exists. The finiteness of $\psi f$ follows as a special case of [23, Theorem 1], where Markov chains in discrete-time on a general state space are considered without any irreducibility assumption, which generalizes an earlier result for ergodic discrete-time Markov chains on a general state space [22, Theorem 1].

Theorem 2. Let $\left(X_{t}\right)_{t \geq 0}$ be an irreducible continuous-time Markov chain with generator matrix $\boldsymbol{Q}=\left(q_{i j}\right)_{i, j \in \delta}, \mathcal{C} \subset \delta$ finite, $\gamma>0$ and let $f, g: \& \rightarrow \mathbb{R}_{\geq 0}$ meet the conditions $(C 1)-(C 3)$. Then $\boldsymbol{Q}$ is regular (it uniquely defines $\left(X_{t}\right)_{t \geq 0}$, the Feller process of $\left.\boldsymbol{Q}\right),\left(X_{t}\right)_{t \geq 0}$ is recurrent, and for any invariant measure $\psi$ the sum $\psi \bar{f}=\sum_{i \in \delta} \psi_{i} f(i)$ is finite.

The regularity and the recurrence follow from [21, Theorem 2.2] so that an invariant measure $\psi$ exists. The finiteness of $\psi f$ can be shown by applying Theorem 1 to the embedded jump chain of $\left(X_{t}\right)_{t \geq 0}$ (cf. [6]). Hence, consider the embedded discrete-time jump chain $\left(Y_{n}\right)_{n \in \mathbb{N}}$ with transition probability matrix $\boldsymbol{P}^{*}=\left(p_{i j}^{*}\right)_{i, j \in \S}$ given by

$$
p_{i j}^{*}=\frac{1}{q_{i}} q_{i j}+\delta_{i j},
$$

where $q_{i}=-q_{i i}$. Since our continuous-time Markov chain $\left(X_{t}\right)_{t \geq 0}$ is irreducible recurrent, the jump chain $\left(Y_{n}\right)_{n \in \mathbb{N}}$ is also irreducible recurrent; see, e.g. [1, pp. 184-188]. An invariant measure $\psi^{*}$ for the jump chain is given by $\psi_{j}^{*}=q_{j} \psi_{j}$. The continuous-time drift condition

$$
\sum_{j=0}^{\infty} q_{i j} g(j) \leq-\gamma f(i)
$$


yields the drift condition

$$
\sum_{j=0}^{\infty} p_{i j}^{*} g(j)-g(i)=\sum_{j=0}^{\infty}\left(\frac{q_{i j}}{q_{i}}+\delta_{i j}\right) g(j)-g(i)=\frac{1}{q_{i}} \sum_{j=0}^{\infty} q_{i j} g(j)=\frac{d_{g}(i)}{q_{i}} \leq-\frac{\gamma f(i)}{q_{i}}
$$

for the embedded chain. Thus, according to Theorem $1, \psi^{*} f^{*}$ with $f^{*}(i)=f(i) / q_{i}$ is finite. Since, obviously, $\psi^{*} f^{*}=\psi f$ the proof is completed.

The following is fundamental for obtaining the state space truncation procedure in the next section.

Theorem 3. Let $\left(Y_{n}\right)_{n \in \mathbb{N}}$ be an irreducible recurrent discrete-time Markov chain with transition probability matrix $\boldsymbol{P}=\left(p_{i j}\right)_{i, j \in s}$, let $\psi$ be an invariant measure, and let $d_{g}=\boldsymbol{P} g-g \leq h$ for some $\psi$-integrable function $h \geq 0$. Then $\psi d_{g}=\psi(\boldsymbol{P} g-g) \geq 0$.

Proof. Without loss of generality let $\delta=\mathbb{N}$. Define

$$
\begin{gathered}
\ell_{i j}^{(n)}:=\mathbb{P}\left(Y_{n}=j, Y_{n-1}, \ldots, Y_{1} \neq i \mid Y_{0}=i\right), \quad i, j \in \mathbb{N}, n \geq 1, \\
\psi_{j}:=\sum_{n=1}^{\infty} \ell_{0 j}^{(n)}, \quad j \in \mathbb{N} .
\end{gathered}
$$

Then for the $\ell_{i j}^{(n)}$, we have the recursion

$$
\ell_{i j}^{(1)}=p_{i j}, \quad \ell_{i j}^{(n)}=\sum_{k \neq i} \ell_{i k}^{(n-1)} p_{k j}, \quad n \geq 2,
$$

which yields

$$
\begin{aligned}
\psi_{j} & =\sum_{n=1}^{\infty} \ell_{0 j}^{(n)} \\
& =p_{0 j}+\sum_{n=2}^{\infty} \sum_{k=1}^{\infty} \ell_{0 k}^{(n-1)} p_{k j} \\
& =p_{0 j}+\sum_{k=1}^{\infty} \sum_{n=1}^{\infty} \ell_{0 k}^{(n)} p_{k j} \\
& =p_{0 j}+\sum_{k=1}^{\infty} \psi_{k} p_{k j} \\
& =\sum_{k=0}^{\infty} \psi_{k} p_{k j}
\end{aligned}
$$

since $\psi_{0}$ is the probability of eventually returning to state 0 , and thus, due to recurrence $\psi_{0}=1$. Therefore, $\psi$ is an invariant measure, and since any other invariant measure is obtained by multiplication with some positive scalar it is sufficient to consider only this particular invariant measure.

Remark 1. In the general case (no recurrence required), by $\left(1, \psi_{1}, \psi_{2}, \ldots\right)$ the minimal subinvariant measure $\psi$ with $\psi_{0}=1$ is defined; see, e.g. [1, pp. 172-174] for more details. 
Now for $N \in \mathbb{N}$ and $j \in\{0, \ldots, N\}$ define

$$
\ell_{0 j}^{(n, N)}:=\mathbb{P}\left(Y_{n}=j, Y_{n-1}, \ldots, Y_{1} \in\{1, \ldots, N\} \mid Y_{0}=0\right), \quad \psi_{j}^{(N)}:=\sum_{n=1}^{\infty} \ell_{0 j}^{(n, N)} .
$$

With similar considerations as above, we have the recursion

$$
\ell_{0 j}^{(1, N)}=p_{0 j}, \quad \ell_{0 j}^{(n, N)}=\sum_{k=1}^{N} \ell_{0 k}^{(n-1, N)} p_{k j}, \quad n \geq 2,
$$

which yields

$$
\begin{aligned}
\psi_{j}^{(N)} & =\sum_{n=1}^{\infty} \ell_{0 j}^{(n, N)} \\
& =p_{0 j}+\sum_{n=2}^{\infty} \sum_{k=1}^{N} \ell_{0 k}^{(n-1, N)} p_{k j} \\
& =p_{0 j}+\sum_{k=1}^{N} \sum_{n=1}^{\infty} \ell_{0 k}^{(n, N)} p_{k j} \\
& =p_{0 j}+\sum_{k=1}^{N} \psi_{k}^{(N)} p_{k j} .
\end{aligned}
$$

Defining $\psi_{j}^{(N)}=0$ for $j>N$ and $\psi^{(N)}=\left(\psi_{j}^{(N)}\right)_{j=0}^{\infty}$, we can state that $\psi_{j}^{(N)}$ increases monotonically in $N$ with $\lim _{N \rightarrow \infty} \psi^{(N)}=\psi$ (componentwise, weak convergence). Now we can consider $\psi^{(N)}(\boldsymbol{P} g-g)$ instead of $\psi(\boldsymbol{P} g-g)$. Since $\psi_{j}^{(N)}=0$ for almost all $j \in \mathbb{N}$, there is no problem when changing the order of summation. We can write

$$
\begin{aligned}
\psi^{(N)} d_{g}= & \psi^{(N)}(\boldsymbol{P} g-g) \\
= & \sum_{i=0}^{N} \psi_{i}^{(N)}\left(\sum_{j=0}^{\infty} p_{i j} g(j)-g(i)\right) \\
= & \psi_{0}^{(N)}\left(\sum_{j=0}^{\infty} p_{0 j} g(j)-g(0)\right)+\sum_{i=1}^{N} \psi_{i}^{(N)}\left(\sum_{j=0}^{\infty} p_{i j} g(j)-g(i)\right) \\
= & \psi_{0}^{(N)} \sum_{j=0}^{\infty} p_{0 j} g(j)-\psi_{0}^{(N)} g(0)+\sum_{i=1}^{N} \psi_{i}^{(N)} \sum_{j=0}^{\infty} p_{i j} g(j)-\sum_{i=1}^{N} \psi_{i}^{(N)} g(i) \\
= & \psi_{0}^{(N)} \sum_{j=0}^{\infty} p_{0 j} g(j)+\sum_{i=1}^{N} \psi_{i}^{(N)} \sum_{j=0}^{\infty} p_{i j} g(j)-\sum_{i=0}^{N} \psi_{i}^{(N)} g(i) \\
= & \psi_{0}^{(N)} \sum_{j=0}^{N} p_{0 j} g(j)+\sum_{i=1}^{N} \psi_{i}^{(N)} \sum_{j=0}^{N} p_{i j} g(j)-\sum_{i=0}^{N} \psi_{i}^{(N)} g(i) \\
& +\sum_{i=0}^{N} \psi_{i}^{(N)} \sum_{j=N+1}^{\infty} p_{i j} g(j)
\end{aligned}
$$




$$
\begin{aligned}
= & \psi_{0}^{(N)} \sum_{j=0}^{N} p_{0 j} g(j)+\sum_{j=0}^{N} g(j) \sum_{i=1}^{N} \psi_{i}^{(N)} p_{i j}-\sum_{i=0}^{N} \psi_{i}^{(N)} g(i) \\
& +\sum_{i=0}^{N} \psi_{i}^{(N)} \sum_{j=N+1}^{\infty} p_{i j} g(j) \\
= & \psi_{0}^{(N)} \sum_{j=0}^{N} p_{0 j} g(j)+\sum_{j=0}^{N} g(j)\left(\psi_{j}^{(N)}-p_{0 j}\right)-\sum_{i=0}^{N} \psi_{i}^{(N)} g(i) \\
& +\sum_{i=0}^{N} \psi_{i}^{(N)} \sum_{j=N+1}^{\infty} p_{i j} g(j) \\
= & \left(\psi_{0}^{(N)}-1\right) \sum_{j=0}^{N} p_{0 j} g(j)+\sum_{i=0}^{N} \psi_{i}^{(N)} \sum_{j=N+1}^{\infty} p_{i j} g(j) \\
\geq & \left(\psi_{0}^{(N)}-1\right) \sum_{j=0}^{N} p_{0 j} g(j) .
\end{aligned}
$$

Due to the finiteness of $d_{g}(0)$ and recurrence, we have

$$
\lim _{N \rightarrow \infty} \sum_{j=0}^{N} p_{0 j} g(j)=d_{g}(0)+g(0)<\infty, \quad \lim _{N \rightarrow \infty} \psi_{0}^{(N)}=1,
$$

and, thus, we obtain

$$
\limsup _{N \rightarrow \infty} \psi^{(N)} d_{g} \geq \lim _{N \rightarrow \infty}\left(\psi_{0}^{(N)}-1\right) \sum_{j=0}^{N} p_{0 j} g(j)=0 .
$$

Due to $d_{g} \leq h$, we have $\psi_{j}\left(h(j)-d_{g}(j)\right) \geq 0$ for all $j \in \mathbb{N}$, and Fatou's lemma yields

$$
\begin{aligned}
\psi\left(h-d_{g}\right) & =\sum_{j=0}^{\infty} \psi_{j}\left(h(j)-d_{g}(j)\right) \\
& =\sum_{j=0}^{\infty} \lim _{N \rightarrow \infty} \psi_{j}^{(N)}\left(h(j)-d_{g}(j)\right) \\
& \leq \liminf _{N \rightarrow \infty} \sum_{j=0}^{\infty} \psi_{j}^{(N)}\left(h(j)-d_{g}(j)\right) \\
& =\liminf _{N \rightarrow \infty} \sum_{j=0}^{\infty} \psi_{j}^{(N)} h(j)-\limsup _{N \rightarrow \infty} \sum_{j=0}^{\infty} \psi_{j}^{(N)} d_{g}(j)
\end{aligned}
$$

Since $h$ is $\psi$-integrable, by monotone convergence, we obtain

$$
\lim _{N \rightarrow \infty} \sum_{j=0}^{\infty} \psi_{j}^{(N)} h(j)=\sum_{j=0}^{\infty} \psi_{j} h(j)=\psi h
$$


and, hence,

$$
0 \leq \psi\left(h-d_{g}\right) \leq \psi h-\limsup _{N \rightarrow \infty} \psi^{(N)} d_{g} \leq \psi h .
$$

From these inequalities, we obtain $h-d_{g}$, and, thus, $d_{g}$ is $\psi$-integrable with $\psi d_{g} \geq 0$.

Now we provide an analogous theorem for continuous-time Markov chains.

Theorem 4. Let $\left(X_{t}\right)_{t \geq 0}$ be an irreducible recurrent continuous-time Markov chain with generator matrix $\boldsymbol{Q}=\left(q_{i j}\right)_{i, j \in s}, d_{g}=\boldsymbol{Q} g \leq h$ for some $\psi$-integrable function $h$. Then $\psi d_{g}=\psi \boldsymbol{Q} g \geq 0$ for any invariant measure $\psi$.

Proof. Consider again the embedded discrete-time jump chain $\left(Y_{n}\right)_{n \in \mathbb{N}}$ with transition probability matrix $\boldsymbol{P}^{*}=\left(p_{i j}^{*}\right)_{i, j \in s}$ given by (2), i.e. $p_{i j}^{*}=\left(1 / q_{i}\right) q_{i j}+\delta_{i j}$, invariant measure $\psi^{*}$ given by $\psi_{j}^{*}=q_{j} \psi_{j}$, drift $d_{g}^{*}$ where $d_{g}^{*}(i)=d_{g}(i) / q_{i}$ (cf. (3)), and upper drift bound $h^{*}$ given by $h^{*}(i)=h(i) / q_{i}$. Obviously, we have $\psi^{*} d_{g}^{*}=\psi d_{g}$, and since $h^{*}$ is $\psi^{*}$-integrable, Theorem 3 yields $\psi^{*} d_{g}^{*} \geq 0$.

Before we apply these results to the task of truncating the state space, we make some remarks concerning the drift bound $h$.

- Under the conditions of Theorem 1 or Theorem 2, respectively, we always have $d_{g}(j) \leq 0$ for all $j \in \delta \backslash \mathcal{C}$, and since $\mathcal{C}$ is finite, $h=d_{g} \mathbf{1}_{\mathcal{C}} \geq d_{g}$ is trivially $\psi$-integrable, where $\mathbf{1}$ is the indicator function.

- A special case of our theorems appears in [12], where positive recurrence and thus the summability of $\psi$ is assumed, and the corresponding drift condition is $\sup d_{g}(j)<\infty$. In this case $h=C=\sup d_{g}(j)$ is, of course, $\psi$-integrable. For a finite-state space $\&$ we trivially could state ' $=0$ ', and ' $\geq 0$ ' is guaranteed by $[12$, Theorem 1(ii)] in a quite general context, where the continuous-time case is given as a special case by [12, Proposition 1].

\section{Truncation of the state space}

Now we exploit the theorems of the previous section in order to determine finite subsets of the state space such that the truncation error is bounded as stipulated by (1). The drift conditions provided by the aforementioned criterion by Tweedie [21, Theorem 2.3] for regularity and positive recurrence have been exploited in [9] in order to obtain bounds of the form $\sum_{i \in \mathcal{C}} \pi_{i} \geq$ $1-\varepsilon$ when approximating the stationary distributions of infinite ergodic continuous-time level dependent quasi-birth-and-death (LDQBD) processes. We will use a similar approach for deriving the desired bounds (1) for recurrent continuous-time and discrete-time Markov chains on countable state spaces. The main idea is to choose $\gamma>0$ and $g$ such that the finite set $\mathcal{C}$ is appropriate for truncation. This procedure is based on the following result.

Theorem 5. Let $f, g, \gamma, \mathcal{C}$ meet the conditions of Theorem 1 or Theorem 2, respectively. Furthermore, let

$$
f(j)>0 \text { for all } j \in \mathcal{C}_{0}:=\left\{i \in \&: d_{g}(i)>0\right\},
$$

and let $f\left(j_{1}\right)>0$ for some $j_{1} \in \overline{\mathfrak{C}}_{0}:=\wp \backslash \mathcal{C}_{0}$. Then, we have

$$
\frac{\sum_{j \notin \mathcal{C}} \psi_{j} f(j)}{\sum_{j \in \mathcal{S}} \psi_{j} f(j)} \leq \frac{c}{c+\gamma},
$$


where

$$
c=\max _{j \in \mathfrak{e}_{0}} \frac{d_{g}(j)}{f(j)}>0 .
$$

Proof. First note that $\mathcal{C}_{0}:=\left\{j \in \delta: d_{g}(j)>0\right\}$ has finitely many elements due to condition (C1). Furthermore, this condition guarantees that $d_{g}\left(j_{1}\right)<0$, yielding $\psi_{j_{1}} d_{g}\left(j_{1}\right)<0$ since the invariant measure $\psi$ has no zero-entry. As pointed out above, by Theorem 3 and Theorem 4 , under the conditions of Theorem 1 or Theorem 2 respectively, we have $\psi d_{g} \geq 0$, and, therefore, there is some $j_{0} \in \mathcal{C}_{0}$ with $d_{g}\left(j_{0}\right)>0$, that is $\mathfrak{C}_{0} \neq \varnothing$. Assumption (4) guarantees that $c>0$ is well defined by (6).

Now we scale $g$ and, thus, $d_{g}$ by $1 /(c+\gamma)>0$, that is

$$
g^{*}(j):=\frac{g(j)}{c+\gamma}, \quad d_{g^{*}}(j)=\frac{d_{g}(j)}{c+\gamma} .
$$

This yields $d_{g^{*}}(j) \leq c f(j) /(c+\gamma)$ for $j \in \mathcal{C}$ and $d_{g^{*}}(j) \leq-\gamma f(j) /(c+\gamma)$ for $j \notin \mathcal{C}$, or, written in concise form

$$
d_{g^{*}}(j) \leq\left(\frac{c}{c+\gamma}-\mathbf{1}_{\overline{\mathcal{C}}}(j)\right) f(j) .
$$

Summation of $\psi_{j} d_{g^{*}}(j)$ yields

$$
0 \leq \psi d_{g^{*}}=\sum_{j \in \mathcal{S}} \psi_{j} d_{g^{*}}(j) \leq \frac{c}{c+\gamma} \sum_{j \in \mathcal{S}} \psi_{j} f(j)-\sum_{j \notin \mathcal{C}} \psi_{j} f(j) .
$$

Due to the assumptions, $\psi f \neq 0$, which immediately implies (5) and completes the proof.

Remark 2. If $f(j)>0$ for infinitely many $j \in \mathcal{S}$, due to the finiteness of $\mathcal{C}$, there will always be some $j_{1} \in \overline{\mathrm{C}}$ with $f\left(j_{1}\right)>0$. If $f(j)>0$ holds only for finitely many $j \in \&$, the truncation of the state space is quite easy, we can simply choose the finite set $\mathcal{C}=\{j: f(j)>0\}$. However, the proof of Theorem 5 can be extended to this case via the obvious inequality

$$
0=\sum_{j \notin \mathcal{C}} \psi_{j} f(j) \leq \frac{c}{c+\gamma} \sum_{j \in \mathcal{S}} \psi_{j} f(j)
$$

where we define $c=0$ for $\mathcal{C}_{0}=\varnothing$ (which is possible in this situation).

With $\varepsilon=c /(c+\gamma)$, Theorem 5 yields the desired bound for our procedure of determining a finite set $\mathcal{C}$ meeting (1). When a Lyapunov function $g$ is given, $c$ is determined by the corresponding drift function $d_{g}$ and, thus, we can only vary $\gamma$. For guaranteeing $c /(c+\gamma)=\varepsilon$, we choose $\gamma=(c / \varepsilon)-c$. Since $\varepsilon<1$ and $c>0$, we have $\gamma>0$. If for this choice of $\gamma$ the set $\mathcal{C}=\left\{j \in \delta: d_{g}(j)>-\gamma f(j)\right\}$ is finite, we have an appropriate truncation of the state space. Otherwise, we have to find a new Lyapunov function. A simple scaling does not help in this case since by the definition of $\gamma$ and $c, \mathcal{C}$ is invariant with respect to scaling of $g$.

The results just derived provide the basis for a method of finding an appropriate set $\mathcal{C}_{0}$ for truncating the sum $\psi f$ as follows.

(i) Choose a Lyapunov function $g$.

(ii) Compute the drift $d_{g}$. 
(iii) Determine

$$
\begin{aligned}
\mathcal{C}_{0} & =\left\{i \in s: d_{g}(i)>0\right\}, \\
c & =\max _{j \in \mathcal{C}_{0}} \frac{d_{g}(j)}{f(j)}, \\
\gamma & =\frac{c}{\varepsilon}-c, \\
\mathcal{C} & =\left\{j \in s: d_{g}(j)>-\gamma f(j)\right\} .
\end{aligned}
$$

(iv) If $\mathcal{C}$ is finite, (1) holds. Otherwise choose a new Lyapunov function and restart with (ii).

\section{Examples}

Now we demonstrate our state space truncation approach by two illustrative examples, where we restrict ourselves to continuous-time Markov chains, as the truncation procedure works similarly in the discrete-time case.

Example 1. We start with an example of a two-dimensional continuous-time Markov chain $\left(X_{t}\right)=\left(X_{t}^{(1)}, X_{t}^{(2)}\right)$ with state space $\delta=\mathbb{N} \times \mathbb{N}$ and transitions according to Table 1 with parameters $\lambda, \mu, \delta_{1}, \delta_{2}>0$, which describes a stochastic gene expression model [20] that was also considered in [9]. As a concrete numerical case, we choose $\lambda=60, \mu=\delta_{2}=0.01$, and $\delta_{1}=0.2$. In [9], the authors looked for a set $\mathcal{C}$ fulfilling

$$
\frac{\sum_{\left(x_{1}, x_{2}\right) \in \mathcal{C}} \pi_{\left(x_{1}, x_{2}\right)}}{\sum_{\left(x_{1}, x_{2}\right) \in \mathcal{S}} \pi_{\left(x_{1}, x_{2}\right)}} \geq 1-\varepsilon,
$$

where $\pi$ is the stationary distribution. The method used in [9] is the above method with $f\left(x_{1}, x_{2}\right)=1$. The Lyapunov function $g$ was defined by

$$
g\left(x_{1}, x_{2}\right)=\left(x_{1}-300\right)^{2}+\left(x_{2}-300\right)^{2},
$$

yielding the drift function

$$
d_{g}\left(x_{1}, x_{2}\right)=-0.4 x_{1}^{2}-0.02 x_{2}^{2}+0.02 x_{1} x_{2}+234.21 x_{1}+6.01 x_{2}-35940 .
$$

Obviously, this drift function is negative up to finitely many values $\left(x_{1}, x_{2}\right)$, the maximum according to (7) is $c=126$, and for $\varepsilon=0.05$, by (8), we have to choose $\gamma=2394$. Thus, from (9), we obtain

$$
\mathcal{C}=\left\{\left(x_{1}, x_{2}\right): d_{g}\left(x_{1}, x_{2}\right) \geq-2394\right\}
$$

as a finite subset of the state space that meets the desired truncation error bound.

It is clear that our truncation procedure does not require a specific transition structure or a specific numbering of the states. In many applications, however, the above characterization of $\mathcal{C}$ might be relatively impractical, in particular when a specific numbering of the states is given and the generator matrix of the Markov chain must be truncated to render numerical computations possible. Think, for example, of infinite LDQBD processes, where the states are ordered according to the chosen level definition and the block structured generator matrix is truncated at certain blocks corresponding to high (or low) level numbers such as, e.g. in [3], [4], [5], [7], [9], and [15]. Then it is often more convenient to consider an appropriate finite superset of $\mathcal{C}$ rather than to work directly with $\mathcal{C}$. For instance, simple algebra yields

$$
\mathcal{C} \subset\left\{\left(x_{1}, x_{2}\right): 221 \leq \max \left\{x_{1}, x_{2}\right\} \leq 657\right\}, \quad \mathcal{C} \subset\left\{\left(x_{1}, x_{2}\right): 250 \leq x_{1}+x_{2} \leq 975\right\},
$$

where the first superset can be found in [9] too. It contains 384123 states, the second one contains 445401 states. 
TABLE 1: State transitions of the gene expression example.

\begin{tabular}{ccc}
\hline From state & To state & Rate \\
\hline$\left(x_{1}, x_{2}\right)$ & $\left(x_{1}+1, x_{2}\right)$ & $\lambda$ \\
$\left(x_{1}, x_{2}\right)$ & $\left(x_{1}, x_{2}+1\right)$ & $\mu x_{1}$ \\
$\left(x_{1}, x_{2}\right)$ & $\left(x_{1}-1, x_{2}\right)$ & $\delta_{1} x_{1}$ \\
$\left(x_{1}, x_{2}\right)$ & $\left(x_{1}, x_{2}-1\right)$ & $\delta_{2} x_{2}$ \\
\hline
\end{tabular}

The Lyapunov function defined above is still appropriate when considering the stationary moments of the first or the second component, respectively, that is, we consider $\mathbb{E}_{\pi}\left[f^{(1)}\right]$ and $\mathbb{E}_{\pi}\left[f^{(2)}\right]$ with $f^{(1)}\left(x_{1}, x_{2}\right)=x_{1}$ and $f^{(2)}\left(x_{1}, x_{2}\right)=x_{2}$, respectively. It is easy to see that

$$
\mathcal{C}_{1}=\left\{\left(x_{1}, x_{2}\right): d_{g}\left(x_{1}, x_{2}\right) \geq-\gamma x_{1}\right\}, \quad \mathcal{C}_{2}=\left\{\left(x_{1}, x_{2}\right): d_{g}\left(x_{1}, x_{2}\right) \geq-\gamma x_{2}\right\}
$$

are finite for arbitrary $\gamma>0$. By simple algebra, we obtain $d_{g}\left(x_{1}, 0\right)<0$ and $d_{g}\left(0, x_{2}\right)<0$ for any $x_{1}, x_{2} \in \mathbb{N}$, yielding that $\mathcal{C}_{0}=\left\{\left(x_{1}, x_{2}\right): d_{g}\left(x_{1}, x_{2}\right)>0\right\}$ does not contain any point $\left(x_{1}, x_{2}\right)$ with $f^{(j)}\left(x_{1}, x_{2}\right)=0$ for $j=1$ or $j=2$. Thus, there is no problem when defining the value $c$ according to (7). For $f^{(1)}$, we have $c=0.42$ and for $\varepsilon=0.05$, by (8), we have $\gamma=7.98$. For simplicity and as a means of comparison, we give supersets for $\mathcal{C}_{1}$ similar to those given above. We have

$$
\mathcal{C}_{1} \subset\left\{\left(x_{1}, x_{2}\right): 231 \leq \max \left\{x_{1}, x_{2}\right\} \leq 665\right\}, \quad \mathcal{C}_{1} \subset\left\{\left(x_{1}, x_{2}\right): 261 \leq x_{1}+x_{2} \leq 993\right\},
$$

where the first superset contains 390195 states and the second one contains 460324 states.

Similarly, for $f^{(2)}$, we have $c \approx 0.4272$ and $\gamma \approx 8.1176$ (for $\varepsilon=0.05$ ), yielding

$$
\mathcal{C}_{2} \subset\left\{\left(x_{1}, x_{2}\right): 230 \leq \max \left\{x_{1}, x_{2}\right\} \leq 920\right\}, \quad \mathcal{C}_{2} \subset\left\{\left(x_{1}, x_{2}\right): 377 \leq x_{1}+x_{2} \leq 1246\right\},
$$

where the first superset contains 795341 states and the second one contains 706875 states.

Example 2. We continue with a simple but extremely instructive example that demonstrates the applicability of the state space truncation procedure to nonergodic recurrent Markov chains and shows some peculiarities with regard to the specific choice of a Lyapunov function.

Consider a birth-death process $\left(X_{t}\right)_{t \geq 0}$ with birth rate $\lambda$ and death rate $\lambda$, that is a Markov chain with state space $\mathbb{N}$ and the generator matrix

$$
\boldsymbol{Q}=\left(\begin{array}{ccccc}
-\lambda & \lambda & & & \\
\lambda & -2 \lambda & \lambda & & \\
& \lambda & -2 \lambda & \lambda & \\
& & \ddots & \ddots & \ddots
\end{array}\right)
$$

Obviously, $\psi=(1,1, \ldots)$ is an invariant measure. Consider the computation of

$$
H=\lim _{t \rightarrow \infty} \frac{\int_{0}^{t}\left(1 /\left(X_{s}+1\right)^{2}\right) \mathrm{d} s}{\int_{0}^{t}\left(1 / 2^{X_{s}}\right) \mathrm{d} s}=\frac{\psi f^{(1)}}{\psi f^{(2)}}, \quad f^{(1)}(j)=\frac{1}{(j+1)^{2}}, \quad f^{(2)}(j)=\frac{1}{2^{j}} .
$$

We want to use our method to find finite sets $\mathcal{C}_{1}$ and $\mathcal{C}_{2}$ such that

$$
\left(1-\varepsilon_{1}\right) H \leq H^{*} \leq \frac{1}{1-\varepsilon_{2}} H,
$$


where

$$
H^{*}=\frac{\sum_{j \in \mathfrak{C}_{1}} \psi_{j} f^{(1)}(j)}{\sum_{j \in \mathfrak{C}_{2}} \psi_{j} f^{(2)}(j)}
$$

is the approximation obtained by finite summation. Obviously,

$$
\frac{\sum_{j \in \mathfrak{C}_{i}} \psi_{j} f^{(i)}(j)}{\sum_{j=0}^{\infty} \psi_{j} f^{(i)}(j)} \geq 1-\varepsilon_{i}, \quad i=1,2
$$

is sufficient. We start with considering $f^{(1)}(j)=1 /(j+1)^{2}, j \in \mathbb{N}$. Since $f^{(1)}(j)>0$ for all $j \in \mathbb{N}$ there are no problems when defining $c=\max _{j \in \mathcal{C}_{0}}\left(d_{g}(j) / f(j)\right)$ according to (7), independent of the Lyapunov function $g$. When directly using Theorem 2 , an appropriate choice for $g$ is

$$
g(j)=\sum_{k=1}^{j+1} \frac{1}{k}, \quad j \in \mathbb{N} .
$$

Then, we have

$$
d_{g}(0)=\frac{\lambda}{2}, \quad d_{g}(j)=-\frac{\lambda}{(j+1)(j+2)}, \quad j=1,2, \ldots
$$

For sufficiently large $j \in \mathbb{N}$ there exists $\gamma>0$ such that $d_{g}(j) \leq-\gamma f(j)$. Therefore, by Theorem 2, $\psi f^{(1)}<\infty$. Now we choose $\gamma$ and $\mathcal{C}$ according to (8) and (9). Hence, $\gamma=\left(c / \varepsilon_{1}\right)-c$, where $c=d_{g}(0)=\lambda / 2$, implying

$$
\mathcal{C}_{1}=\left\{j \in \delta:-\frac{\lambda}{(j+1)(j+2)}>-\left(\frac{\lambda}{2 \varepsilon_{1}}-\frac{\lambda}{2}\right) \frac{1}{(j+1)^{2}}\right\} .
$$

It is straightforward to see that $\mathcal{C}_{1}$ is a finite set if and only if $\varepsilon_{1}>\frac{1}{3}$, which for reasonable $\varepsilon_{1}$ is, of course, not true. Therefore, we have to find a new Lyapunov function. Choose

$$
g(j)=\sum_{k=1}^{j+1} \frac{1}{\sqrt{k}}, \quad j \in \mathbb{N} .
$$

Then, we have $c=d_{g}(0)=\lambda / \sqrt{2}$ and

$$
d_{g}(j)=-\frac{\lambda}{\sqrt{(j+1)(j+2)}(\sqrt{j+1}+\sqrt{j+2})}, \quad j=1,2, \ldots
$$

Since $d_{g}(j) \approx \lambda / 2 j^{3 / 2}$ for large $j$,

$$
\mathcal{C}_{1}=\left\{j:-\frac{\lambda}{\sqrt{(j+1)(j+2)}(\sqrt{j+1}+\sqrt{j+2})}>-\left(\frac{\lambda}{2 \varepsilon_{1}}-\frac{\lambda}{2}\right) \frac{1}{(j+1)^{2}}\right\}
$$

is finite for any $\varepsilon_{1}>0$. For $\varepsilon_{1}=\frac{1}{20}$, we obtain $\mathcal{C}_{1}=\{0,1, \ldots, 361\}$.

Now consider $f^{(2)}(j)=1 / 2^{j}, j \in \mathbb{N}$. Our first Lyapunov function $g$, defined by

$$
g(j)=\sum_{k=1}^{j+1} \frac{1}{k}, \quad j \in \mathbb{N}
$$


is also appropriate for $\psi f^{(2)}<\infty$ by Theorem 2 . In this case, this Lyapunov function can be used for defining $\mathcal{C}_{2}$ since

$$
\mathcal{C}_{2}=\left\{j \in \delta:-\frac{\lambda}{(j+1)(j+2)}>-\left(\frac{\lambda}{2 \varepsilon_{2}}-\frac{\lambda}{2}\right) \frac{1}{2^{j}}\right\}
$$

is finite for all $\varepsilon_{2}>0$. For $\varepsilon_{2}=\frac{1}{20}$, we obtain $\mathcal{C}_{2}=\{0, \ldots, 10\}$.

Note that since, for this example, we know the exact invariant measure $\psi=(1,1,1, \ldots)$ and $\psi f^{(1)}=\pi^{2} / 6$, we can easily determine the 'best choice' for $\mathcal{C}_{1}$, namely $\mathcal{C}_{1}=\{0,1, \ldots$, 11\}. Similarly, from $\psi f^{(2)}=2$, we know that $\mathcal{C}_{2}=\{0, \ldots, 4\}$ would be the best choice.

Hence, the example demonstrates that there are Lyapunov functions that meet the conditions of Theorem 2 but are not suitable for our state space truncation procedure. Additionally, we see that the truncations we obtain are quite conservative. This implies that the truncation errors are actually much smaller than requested. This can be interpreted as an advantage, but we also have to consider that usually we have to solve for $\psi$ and/or $\psi f$ numerically, implying that conservative truncations imply higher effort. Of course, tight bounds are desirable.

\section{Conclusion}

With regard to long-run averages of additive functionals in infinite recurrent Markov chains, we have exploited Foster-Lyapunov-type drift conditions in order to obtain finite subsets of the infinite-state space such that at most a prescribed (small) portion of the long-run average lies outside this finite set. This can be taken as a state space truncation method with bounded truncation error, which is extremely useful for, e.g. numerically computing long-run averages, where a state space truncation is inevitable. The approach is independent of specific ways of computing long-run averages. In either case it provides a bound on the approximation error due to the state space truncation. Error bounds for long-run averages rather than for probabilities are particularly valuable when we have a method available that computes long-run averages without explicitly relying on the stationary distribution (if it exists) or an invariant measure. In particular, the state space truncation method solves the open issue that the memory-efficient matrixanalytic method presented in [5] for computing stationary expectations in LDQBD processes without at first explicitly computing the stationary distribution was lacking an accuracy measure. Now, in conjunction with the state space truncation method of this paper, [5] constitutes a powerful matrix-analytic method for numerically approximating long-run averages of additive functionals in infinite recurrent LDQBD processes, where an approximation error bound can be specified a priori. This enormously advances the state of the art in matrix-analytic computations and their applicability to, e.g. performance analysis of complex networks with infinite multidimensional state spaces. Moreover, as the state space truncation method is not restricted to Markov chains with a specific transition structure, it provides many new options for the analysis of a large class of stochastic models.

A couple of further research issues arise. We have considered nonnegative functions $f$, which makes sense, since by applying generalized ergodic theorems we have to guarantee the finiteness of $\psi|f|$. In many applications, nonnegative functions are indeed sufficient to model the problem at hand. Nevertheless, further research on generalizations to arbitrary functions $f$ is 
desirable and currently ongoing. For the tightness of the approximation error bounds, the chosen Lyapunov function is a crucial factor. Not all Lyapunov functions that guarantee the finiteness of the long-run average under consideration are suitable for our state space truncation method; some lead to infinite subsets. Furthermore, even if suitable, different Lyapunov functions yield different finite subsets corresponding to different tightness of the respective bounds. Hence, the systematic derivation of Lyapunov functions that are good in the sense of yielding as tight bounds as possible deserve further attention. For instance, restricted function classes might be considered as candidate Lyapunov functions and their properties with regard to the state space truncation method are to be studied.

\section{References}

[1] Anderson, W. J. (1991). Continuous-Time Markov Chains. Springer, New York.

[2] Asmussen, S. (2003). Applied Probability and Queues, 2nd edn. Springer, New York.

[3] Baumann, H. and Sandmann, W. (2010). Numerical solution of level dependent quasi-birth-and-death processes. Procedia Comput. Sci. 1, 1561-1569.

[4] Baumann, H. and Sandmann, W. (2012). Steady state analysis of level dependent quasi-birth-and-death processes with catastrophes. Comput. Operat. Res. 39, 413-423.

[5] Baumann, H. and Sandmann, W. (2013). Computing stationary expectations in level-dependent QBD processes. J. Appl. Prob. 50, 151-165.

[6] Baumann, H. and Sandmann, W. (2014). On finite long run costs and rewards in infinite Markov chains. Statist. Prob. Lett. 91, 41-46.

[7] Bright, L. AND TAYLOR, P. G. (1995). Calculating the equilibrium distribution in level dependent quasi-birthand-death processes. Commun. Statist. Stoch. Models 11, 497-525.

[8] Chung, K. L. (1960). Markov Chains with Stationary Transition Probabilities. Springer, Berlin.

[9] Dayar, T., Sandmann, W., SpIeler, D. And Wolf, V. (2011). Infinite level-dependent QBD processes and matrix-analytic solutions for stochastic chemical kinetics. Adv. Appl. Prob. 43, 1005-1026.

[10] Foster, F. G. (1953). On the stochastic matrices associated with certain queueing processes. Ann. Math. Statist. 24, 355-360.

[11] Gibson, D. And Seneta, E. (1987). Augmented truncations of infinite stochastic matrices. J. Appl. Prob. 24, 600-608.

[12] Glynn, P. W. And Zeevi, A. (2008). Bounding stationary expectations of Markov processes. In Markov Processes and Related Topics: A Festschrift for Thomas G. Kurtz (Inst. Math. Statist. Collect. 4), Institute of Mathematical Statistics, Beachwood, OH, pp. 195-214.

[13] Golub, G. H. and Seneta, E. (1973). Computation of the stationary distribution of an infinite Markov matrix. Bull. Austral. Math. Soc. 8, 333-341.

[14] Golub, G. H. And Seneta, E. (1974). Computation of the stationary distribution of an infinite stochastic matrix of special form. Bull. Austral. Math. Soc. 10, 255-261.

[15] Hanschke, T. (1999). A matrix continued fraction algorithm for the multiserver repeated order queue. Math. Comput. Modelling 30, 159-170.

[16] Latouche, G. AND Taylor, P. (2002). Truncation and augmentation of level-independent QBD processes. Stoch. Process. Appl. 99, 53-80.

[17] Pakes, A. G. (1969). Some conditions for ergodicity and recurrence of Markov chains. Operat. Res. 17, 1058-1061.

[18] Seneta, E. (1981). Nonnegative Matrices and Markov Chains, 2nd edn. Springer, New York.

[19] Serfozo, R. (2009). Basics of Applied Stochastic Processes. Springer, Berlin.

[20] Thattai, M. and van Oudenaarden, A. (2001). Intrinsic noise in gene regulatory networks. Proc. Nat. Acad. Sci. USA 98, 8614-8619.

[21] Tweedie, R. L. (1975). Sufficient conditions for regularity, recurrence and ergodicity of Markov processes. Math. Proc. Camb. Phil. Soc. 78, 125-136.

[22] Tweedie, R. L. (1983). The existence of moments for stationary Markov chains. J. Appl. Prob. 20, $191-196$.

[23] Tweedie, R. L. (1988). Invariant measures for Markov chains with no irreducibility assumptions. A Celebration of Applied Probability (J. Appl. Prob. Spec. Vol. 25A), Applied Probability Trust, Sheffield, pp. 275-285.

[24] Tweedie, R. L. (1998). Truncation approximations of invariant measures for Markov chains. J. Appl. Prob. 35, 517-536.

[25] Zhao, Y. Q. And Liu, D. (1996). The censored Markov chain and the best augmentation. J. Appl. Prob. 33, 623-629. 